\title{
Las políticas de género en una universidad pública estatal: discrepancias institucionales
}

Sergio-Alberto López-Molina y Marina Vázquez-Guerrero

\section{RESUMEN}

Este artículo presenta un análisis de la documentación institucional sobre políticas de género en una universidad pública mexicana y las acciones concretas en la gestión cotidiana. Tras la revisión detallada de normativas, códigos y políticas institucionales que funcionan como marcos de referencia para la práctica educativa en una universidad pública, se contrastó con el testimonio de algunos directivos de alto nivel, permitiendo conocer cómo estos referentes intervienen en las relaciones estructurales. Entre los principales hallazgos se puede identificar que la preocupación por temas como la violencia de género es reciente y sólo se ha trabajado en el nivel de investigaciones de cuerpos académicos, sin llegar a influir en el nivel institucional para la toma de decisiones, por lo tanto, los temas de género no son prioritarios y cuando se incluyen se ponen en la misma jerarquía de grupos vulnerables.

Palabras clave: género, universidades públicas, análisis institucional, cultura institucional, México.

\section{Sergio-Alberto López-Molina}

sergio_lopez@ucol.mx Mexicano. Doctor en Educación por la Universidad de Colima, México. Profesor-investigador de la Facultad de Psicología de la Universidad de Colima. Temas de investigación: sujetos e instituciones educativas. 


\section{As políticas de gênero em uma universidade pública estatal: discrepâncias institucionais}

\section{RESUMO}

Este artigo apresenta uma análise da documentação institucional sobre políticas de gênero em uma universidade pública mexicana e as ações concretas na gestão cotidiana. Depois da revisão detalhada de normativos, códigos e políticas institucionais que funcionam como marcos de referência para a prática educativa em uma universidade pública, se contrastou com o testemunho de alguns diretivos de alto nível, permitindo conhecer como estas referencias intervém nas relações estruturais. Entre as principais descobertas se pode identificar que a preocupação por temas como a violência de gênero é recente e só se tem trabalhado no nível de pesquisas de corpos acadêmicos, sem chegar a influir no nível institucional para a tomada de decisões, por tanto, os temas de gênero não são prioritários e quando se incluem se põem na mesma hierarquia de grupos vulneráveis.

Palavras chave: gênero, universidades públicas, análise institucional, cultura institucional, México.

\section{Gender policies in a state public university: institutional discrepancies}

\section{ABSTRACT}

This article presents an analysis of the institutional documentation on gender policies in a Mexican public university and the concrete actions in day-to-day management. After a detailed review of regulations, codes and institutional policies that are used as reference frameworks for educational practice in a public university, the author contrasted it with the testimony of some high-level managers, allowing us to know how these referents intervene in structural relationships. Among the main findings we can identify that the concern for issues such as gender violence is recent and has only been worked at at the level of research by academic bodies, without influencing the institutional decision-making level, which means that in effect gender issues are not a priority and when they are included they are placed in the same hierarchy as other vulnerable groups.

Key words: gender, public universities, institutional analysis, institutional culture, Mexico.

Recepción: 11/04/16. Aprobación: 08/08/17. 


\section{Introducción}

Este documento forma parte de la investigación "Análisis de las prácticas discursivas de discriminación y violencia simbólica de género que intervienen en el espacio educativo universitario: de las políticas institucionales a las conversaciones cotidianas" y tiene por objetivo llevar a cabo una revisión documental sobre la existencia y características de normativas, códigos y políticas institucionales relacionadas con el género que funcionan como marcos de referencia para la práctica educativa en una universidad pública mexicana, ${ }^{1}$ sin embargo, el interés del estudio no es enfocado al caso, sino a la situación que posiblemente sea común en el contexto nacional.

Una vez que se analizaron los discursos de género que contienen los documentos, se contrastaron con la interpretación y los usos prácticos que hacen los directivos educativos de alto nivel, lo que permite conocer cómo estos referentes intervienen en las relaciones estructurales de género en la universidad. Para lograrlo, se entrevistó a dos directivos relacionados con las problemáticas cotidianas de escuelas y facultades con el fin de conocer su percepción e interpretación de la violencia y equidad de género en la institución.

Estos resultados permitieron analizar la manera en que las normativas, códigos y políticas institucionales son interpretados y puestos en práctica por estudiantes y profesores universitarios.

\section{El género desde una perspectiva institucional}

Las instituciones son formas sociales que definen las condiciones concretas de existencia de las personas, tanto en el plano material, como en el concerniente a la generación, propagación y difusión del sistema de normas, valores, prácticas y actitudes compartidas por la sociedad o sectores específicos de ésta, todo lo cual sirve de marco de referencia a las decisiones y acciones que los individuos realizan en torno a diferentes aspectos que rodean su reproducción (Lourau, 2001).

Las instituciones son proveedoras de protección de los sujetos que pertenecen a ellas, quienes acatan las normas tendrán beneficios y el cobijo de los otros sujetos que integran la institución; sin embargo, los sujetos que no respeten las disposiciones serán castigados e incluso excluidos de ésta. En las relaciones entre los géneros, las instituciones permiten los procesos de transferencia que se aprehenden en las actividades cotidianas, con la utilización de conceptos cotidianos sobre la división entre lo masculino y lo femenino, estructurando la percepción y la organización de toda una vida social (Lamas, 2000).

En las instituciones, los hábitos y los modos de comportamiento se ven rigidizados a lo largo del tiempo, ligados a la conservación y autoconservación de la institución. También se da una rigidez de los roles, con un conjunto de proyecciones frecuentemente mantenidas en complicidad, que tienden a aprisionar a los sujetos en representaciones coaguladas (Remedi, 2004). Los sujetos, en el afán de proteger a la institución, realizan funciones repetitivas sedimentadas en los roles, como una forma de reintegrar el cobijo prestado por ésta; así se encuentran formas de ocultar o minimizar aquellos aspectos que ante ojos de los no iniciados podrían ponerla en riesgo. Son prácticas "defensivas" y "protectivas" empleadas para salvaguardar la impresión fomentada por un sujeto ante la presencia de otros y por sobre todo de otros de fuera (Goffman, 2012). Una parte se muestra y otra se esconde, sólo puede ser leída entre líneas o percibida cuando existe una larga exposición en tiempo a la dinámica.

La universidad es una institución por su permanencia en el tiempo y sus esquemas estructuradores de comportamiento de los sujetos que pertenecen a ella. Desde la perspectiva de Foucault (2010), en las

\footnotetext{
${ }^{1}$ Institución pública fundada en 1940. Forma parte de las universidades afiliadas a la ANUIES. Se ubica en el occidente de México.
} 
instituciones se refleja un fuerte ejercicio de poder. En el caso de la universidad, como institución, tiene la función de dejar al estudiante fuera de circulación, neutralizando por y para la sociedad a los jóvenes de 18 a 25 años mediante una exclusión forzada, al margen de la sociedad, relegado a un campus, en un recinto donde se construye a su alrededor una sociedad artificial con mecanismos sociales ficticios. Para que una vez que el estudiante ha pasado seis o siete años de su vida en esta sociedad artificial re-integrarlo, desde una perspectiva consumista, es un sujeto asimilable que por lo tanto la sociedad ya puede consumirlo (ibidem: 369 ).

Las universidades como instituciones productoras, reproductoras y controladoras de la sociedad incorporan un esquema pedagógico que colabora a la transferencia de los aspectos patriarcales de control, en donde se fomenta la superioridad del hombre sobre la mujer o se educa a la mujer para que se someta a las instrucciones de los hombres.

Para logarlo, las instituciones se caracterizan por crear un sistema de poder, no solo económico sino también político (Foucault, 2010). Las personas que dirigen las instituciones se atribuyen el derecho a dar órdenes, el derecho a establecer reglamentos, a adoptar medidas, a expulsar unos individuos y aceptar otros. Así, este mismo poder político y económico es también un poder judicial: se dispone del derecho de castigar y recompensar, se evalúa, se clasifica, se dice quién es el mejor y quién es el peor. En este mismo esquema judicial entran las dinámicas que fomentan las inequidades de género para las mujeres universitarias, tanto para estudiantes como para el personal docente y administrativo. De esta forma, el sexismo en las instituciones educativas se afirmó como una necesidad, al atribuir a cada sexo un cometido social preescrito.

Si la educación tenía como fin educar para formar individuos (hombres) autónomos, con las mujeres la discusión era cpara qué molestarse en discutir sobre personas cuyo destino sería la dependencia? Este discurso justificó que se les cerraran las puertas de las aulas a las mujeres y la causa de que hayan padecido condiciones de discriminación en término de oportunidades educativas (Miranda, 2007).

Fue a partir de los años ochenta cuando la cobertura en educación superior se incrementó permitiendo un mayor acceso de matrícula femenina en las universidades, la cantidad de mujeres que acceden a la educación superior es muy similar a la de los varones, por tanto, las tasas de escolaridad de mujeres y hombres fueron semejantes. Durante un par de décadas los estudios se enfocaron a la denuncia de las desigualdades entre hombres y mujeres en la universidad, en su mayoría de carácter cuantitativo, basados en encuestas nacionales o en análisis de indicadores sociales obtenidos de diversos censos, informes, etcétera.

En los últimos años se empezó a explotar la vertiente cualitativa en tratados acerca de las mujeres acercándose más a la fase de "recuperación de la voz", lo que implica el rescate de sus saberes, conocimientos y perspectivas, al reconocer el valor que tienen para la vida humana, para un conocimiento más complejo de la realidad (Martínez, 2006).

En la actualidad, el debate ya no está en el acceso sino en si la educación para las mujeres y los varones sigue reproduciendo los estereotipos de género. Así, los trabajos sobre género empezaron a preocuparse por las desigualdades cualitativas, destacando la persistencia de formas de exclusión y discriminación más sutiles (Miranda, 2007).

\section{El problema}

En la dinámica cotidiana se disimulan fácilmente los ejercicios de poder político, económico y judicial ejercido hacia las mujeres en las universidades, debido a que es un asunto sutil, cotidiano, que se disfraza entre el supuesto cobijo de la institución y forma parte de una cultura que se produce y reproduce en las distintas áreas del conocimiento, y que además es poco cuestionado tanto por hombres como por 
mujeres, lo que representa una gran capacidad de transferencia y reproducción de los roles de género; donde la labor del docente ejerce una influencia en las identidades institucionales y personales: su (re) construcción y (re)producción (Luke, 1999).

En el caso de los y las estudiantes, cuando logran reconocer una injusticia y exponerla, es común que los profesores frenen las inconformidades, modificando las percepciones - increíblemente- con argumentos simples como un "así ha sido siempre", o "así lo hacen todos" y entonces la insurgencia instituyente es callada, eliminada o reprimida, generando un sentimiento de inconformidad y de impotencia ante la injusticia y las pocas posibilidades de intervenir, a lo que tarde o temprano prefieren acostumbrarse ante el sentimiento de impotencia y aún llegar a considerarlo "algo normal". Esta situación es aún más notoria en aquellas carreras tradicionales que tienen una amplia trayectoria e identidad profesional.

La exclusión y falta de equidad entre hombres y mujeres en las universidades ocurre en situaciones cotidianas, que representan el día a día, pero que se perciben como normales: los hombres hablan y controlan durante periodos más largos en clase, interrumpen a las mujeres con comentarios triviales para menospreciar y demostrar la insignificancia de sus participaciones en clase (Ruiz y Ayala, 2016), en suma son microconductas acumulativas de humillación dirigidas contra las mujeres (Lee, 1999).

Los acercamientos sucesivos al problema implican identificar y sortear las capas de complejidad que lo cubren, es decir, niveles de sedimentación que protegen el orden establecido. La posibilidad de "encubrir", representa un primer nivel de complejidad al analizar el tema de género desde una perspectiva institucional, por ello Foucault (2010) propone comprender la sociedad y la civilización a partir sus sistemas de ocultamiento, sus formas de rechazo, de negación, a través de lo que no se quiere, a través de sus límites, del sentimiento de obligación que incita a suprimir un determinado número de cosas, de personas, de procesos; a través, por tanto, de lo que se deja oculto bajo el manto del olvido, en fin, analizando los sistemas de represión-eliminación propios de la sociedad.

Desechamos las palabras de los niños, las personas ebrias, los "locos", los poetas, y posiblemente se traten de forma similar las palabras pronunciadas por las mujeres (Morgan, 1999). Por ello se busca mostrar y analizar lo dicho en el discurso, por hombres y mujeres, con la finalidad de darles voz y descubriendo aquello que mediante represión se intenta eliminar, obviar o restarle importancia.

Las funciones al interior de la institución no siempre operan en un nivel de complementariedad, apoyo recíproco, sino que las funciones oficiales y secundarias pueden estar en conflicto, contrarrestando y oponiendo resistencia a las normas o acciones emprendidas o establecidas por otras instituciones (Fernández, 2005). Los discursos de género que participan en la vida institucional representan un conjunto de fuerzas en lucha permanente al interior de ésta; una constante confrontación entre el ser y el deber ser, entre lo instituyente y lo instituido.

Abordar el tema de la violencia simbólica de género en las instituciones es toparse con frases diluidas que se manifiestan en el día a día: "Lo que ocurre es normal", "Así ha sido siempre". Se transforman a su vez en discursos que se rigidizan, se encarnan y definen hábitos de comportamiento. Existe una negación ante esta situación. Las actitudes al respecto son las de ocultarlo o negarlo ante la percepción de la injusticia y la impotencia para que las situaciones cambien. Es una dinámica de minimización del problema, con las complicidades y acuerdos imperceptibles entre víctimas y victimarios, que se legitiman en los roles de género transmitidos y reproducidos en la búsqueda por conservar la hegemonía masculina (Palomar, 2011).

La situación descrita nos permite reconocer que los movimientos en las instituciones -incluyendo las educativas - , son poco perceptibles, se avanza, pero no a pasos agigantados. Así, desde la perspectiva de 
"lo institucional" es que se abordarán las normativas, códigos y políticas institucionales de la institución analizada, pero no solamente las vigentes, sino que hacemos un recorrido histórico que nos permita localizar algunas coordenadas que finalmente dibujen ciclos que se repiten, y otros más que se eliminen o sustituyan. Indagar los estilos y las dinámicas nos remite de forma inmediata a la búsqueda de la "institución", su cultura y tradiciones que normalizan la conducta y administran la vida de los sujetos.

\section{El método}

En las investigaciones sobre género, una de las tendencias es comparar las dinámicas entre hombres y mujeres respecto a una "diferencia natural" que opera como si hubiera una realidad objetiva que estableciera una diferencia sexual de la mujer con respecto al hombre, tal como lo establece la naturaleza o la biología (Eisenstein, 1999).

Entonces, para comprender las interacciones sociales al interior de la universidad, es necesario comprender el esquema que se rige en la cultura institucional con respecto al género. Esto representa un nivel de complejidad, un esquema de "ocultación" institucionalizado, pues tal y como lo retoma Lamas de Bourdieu (1997), las personas tienen cierto interés en no comprender, desconocer, e ignorar de manera voluntaria los significados de la cultura en la que viven y por lo tanto no comprender algunas cuestiones de su vida cotidiana, con lo que se colabora al mantenimiento y reproducción de un orden social (Lamas, 2000).

El caso seleccionado es una universidad pública estatal ubicada en el occidente de México, está conformada por 65 planteles distribuidos en la totalidad de los municipios del estado. Para 2016, la planta docente estaba conformada por 1949 académicos. La matrícula estaba formada por un total de 27444 alumnos de los cuáles 13931 son de nivel medio superior, 12868 de licenciatura, mientras que 645 realizan estudios de posgrado. En términos porcentuales, las mujeres representan el 53.1\%, mientras que los hombres el 46.9\% (Informe Rectoría, 2016).

Una vez definido el caso se realizó la búsqueda del discurso sobre equidad de género en algunos documentos de la universidad seleccionada: los informes de labores de los rectores, los planes institucionales de desarrollo, el reglamento escolar vigente y la planeación realizada para programas de apoyo federales, todo esto con la finalidad de indagar sobre la presencia de acciones en favor de la equidad de género que se previeron a lo largo de las distintas etapas de la historia de la institución elegida.

Con este análisis se obtiene el "deber ser", pero resulta necesario complementar la información, para la cual se realizaron entrevistas a directivos clave para obtener datos que ayudan a conocer "cómo es", es decir, la forma en que se traducen y decantan las acciones consideradas en la planeación, la forma en que los sujetos adaptan la normativa, así como descubrir los aspectos cotidianos de la vida institucional que no se refleja en los informes rectorales.

Así, para analizar la apropiación de los aspectos normativos y de la perspectiva de género, se entrevistó a dos funcionarios de alto nivel en la universidad, uno, encargado del programa institucional de desarrollo y capacitación del personal académico y otro, responsable del programa institucional de tutoría de los alumnos. La importancia de ambos directivos está dada por su ámbito de influencia tanto con la totalidad de la planta docente, como con el alumnado. Por motivos de confidencialidad, no se revelarán aspectos personales ni laborales de las personas entrevistadas.

Para estructurar la guía de las entrevistas se consideraron en la primera sección la percepción de la decantación de los programas nacionales en educación superior para disminuir la violencia de género y la discriminación de género, así como el fomento de la equidad de género y la influencia que redunda en cambios para la institución y en programas que la misma universidad implementa. 
En un segundo apartado de la guía, se aborda la presencia de casos de violencia de género, inequidad de género y discriminación por identidades de sexo y género. El tercer apartado aborda temas específicos en los que se pregunta sobre comités de vigilancia, la investigación de los temas de violencia, equidad y discriminación por identidades de sexo y género, y las formas en que se denuncian por parte de los trabajadores y el alumnado.

Esta guía fue pensada para una entrevista semiestructurada, que permitiera abundar en conceptos y dinámicas mencionados, con el objetivo de recuperar una "descripción densa"(Geertz, 2005): rescatar "lo dicho" en el discurso perecedero y fijarlo en términos susceptibles de consulta, abordado en contextos pequeños (microscópicos), de forma detallada y profunda (más allá de lo obvio y superficial), presentando la multiplicidad de estructuras conceptuales complejas de un fenómeno social y acorde al planteamiento del problema de género desde una perspectiva institucional.

Se utilizó el Análisis Crítico del Discurso (Iñiguez, 2003; Wodak y Meyer, 2003) para las entrevistas, a partir del cual se realizó el estudio del significado, de referencia e interpretación de palabras y frases específicas que se consideraron de valor interpretativo. $\mathrm{Al}$ respecto, se consideró que el discurso no solamente expresa sino que también construye y confirma prejuicios y de esa manera contribuye a su reproducción y por lo tanto juega un papel crucial en la expresión y la (re)producción de cogniciones sociales que como miembros de grupo comparten regulando y controlando actos e interacciones (Van Dijk, 2002).

En definitiva, se buscó descifrar y desentrañar cómo es la comunicación e interacción en niveles formales e informales encargados de la distribución del poder, el control que las hace perdurar y una descripción de la forma como se dan las dinámicas institucionales con respecto al género: sus orígenes y la forma en como han mutado a lo largo del tiempo, lo que permitiría generar propuestas de cambio pertinentes y relevantes, pero por sobre todo viables, tanto en recursos como políticamente.

\section{La institución y su contexto en la equidad de género}

La investigación sobre "lo institucional" debe ser, por naturaleza propia, la búsqueda de las transformaciones a lo largo del tiempo, debido a que es el pasado de la misma lo que configura las condiciones actuales, por lo tanto, el investigador está obligado a desentrañar la conformación de la institución en sus aspectos socioculturales, sus cuestiones históricas, los niveles de dependencia/interacción de sujetos, la forma en que los líderes llegaron a serlo, entre otras (Elías, 2009). Por lo anterior, para analizar el establecimiento institucional deben considerarse periodos largos, prestando atención a lo histórico para determinar cuáles son las dinámicas de reciente aparición, y cuáles ya existían y simplemente no se les había prestado atención.

A continuación se analizan algunos puntos nodales de la historia institucional de la institución elegida, aclarando que no existe la creencia de que antes y después de estos periodos existía una total tranquilidad y reposo, donde la institución se comporta como un ente estático - en una posición de equilibrio- (Elías, 2009), por el contrario, se afirma que la universidad se mantiene en movimiento continuo, siempre en desplazamiento.

La institución elegida ha transitado por distintas etapas. Su línea de tiempo supera ya las siete décadas, en las cuales se distinguen tres momentos: la germinación (1940-1962), primeros brotes (1962-1981), crecimiento y desarrollo (1980-1997); que se configuran a partir de cuatro eventos clave: fundación, conquista de autonomía universitaria, descentralización y certificación de la calidad (Monroy et al., 2010).

En el periodo rectoral 1981-1985 que la Universidad tiene un notable impulso en el crecimiento de la oferta educativa, el apoyo a la investigación y por tanto en el aumento en la matrícula y la infraestructura, 
así como en la contratación de recién egresados para atender la demanda educativa. La masificación abrió las puertas a mujeres que deseaban tener estudios de licenciatura en la ciudad. La inserción de las mujeres en la educación superior es un hecho reciente (Rodríguez, 2008), sin embargo, el ingreso de más mujeres a la educación superior no eliminó los aspectos de feminización de las carreras, pues el ingreso de las mujeres no se daba en carreras masculinizadas como las ingenierías. Para 1981 las mujeres representaban el 11.7\% de las áreas de ingeniería y tecnología, cifra que aumentó al 22.8\% en 1990 (Martínez, en Acuña, 2008), aun cuando las mujeres representaban ya el $46 \%$ del total de la matrícula institucional.

Durante el periodo rectoral 1989-1997 existió un fuerte impulso a los aspectos de equidad de género en la institución. En primer término, en 1993 se crea la Asociación Colimense de Universitarias (ACU) con más de 100 mujeres profesionistas que querían adentrarse en el campo de los estudios de género. Posteriormente, este mismo grupo, con el visto bueno y apoyo del rector, crea una revista, que posteriormente se llamaría Géneros. Por último, se creó el Centro Universitario de Estudios de Género y, en el nicho de éste, el Diplomado en Estudios de Género. En este periodo las universitarias van tomando posiciones y participando en acciones para abrir espacios de investigación, divulgación y promoción del enfoque de género (Acuña, 2008).

Hasta este periodo rectoral resulta complicado obtener datos que sean relevantes sobre las transiciones por las que han pasado los temas de género en la institución. En un seguimiento realizado por Acuña (2008) a los distintos informes rectorales, afirma que el lenguaje utilizado hace referencia al "cuerpo docente", con lo que aparenta que la composición fuera totalmente masculina, pero no hacen referencia al número de profesoras, ni administrativas, secretariales o servicio. El asunto no sólo redunda sobre ser o no mencionado, o que en el discurso se pretenda ser neutral, sino que también dificulta rastrear la participación de las mujeres durante el periodo de crecimiento y desarrollo de esa universidad.

El énfasis en lo cuantitativo sería lo que permearía los periodos rectorales de 1997-2005 y 2005-2013, en respuesta a la estructura planteada por la Secretaría de Educación Pública (SEP) para el presupuesto de la educación superior, donde la asignación de recursos es no regularizable, mediante proyectos en donde el recurso se otorga a partir del aumento de indicadores sin prestar atención a temas de género y equidad que garanticen para las mujeres su ingreso, permanencia y egreso en educación superior.

Para el periodo rectoral 2013-2017, se comienza a considerar a la presidencia de la ACU como un sector importante, junto con el presidente de la Federación de Estudiantes, el líder sindical, el líder de los jubilados y pensionados y la directora de la Federación de Egresados de la institución. Es pertinente mencionar que de los pilares institucionales los dos menos significativos son los que ocupan las mujeres, mientras que los de mayor influencia y poder político (el sindicato y la Federación de Estudiantes) lo ocupan hombres.

En esta corta revisión de aspectos institucionales resalta el hecho de que son las asociaciones de mujeres alrededor de la universidad las que pugnan por la equidad de género, más no los rectores. Destaca, además, que la preocupación por la agenda de género varía en cada periodo rectoral, situación que impide un crecimiento constante en los esfuerzos. En apariencia, los rectores apoyan a estas mujeres organizadas, más con miras políticas que por convencimiento de la necesidad de lograr la equidad de género en las universidades.

Como puede observarse en los estudios de Acuña (2008), los informes de los rectores son una fuente de datos que ya ha sido utilizada para su interpretación, representando de esta manera una presencia, sin embargo es poco lo que se menciona respecto a los procesos de planeación y los proyectos que proponen estrategias para impulsar los aspectos de género en la 
institución, se detecta una ausencia en los temas de la visión de futuro de los temas de género, es por ello que en el siguiente apartado se analiza lo propuesto en planes institucionales de desarrollo y proyectos con apoyos financieros específicos y las acciones que en ellos se plantean.

\section{Análisis de los Planes Institucionales de Desarrollo}

En la búsqueda realizada en los planes institucionales de desarrollo (PIDE) destaca que el tema de equidad de género se presenta en términos de equidad de ingreso, permanencia y egreso, sin embargo las estrategias para lograr la equidad de género no aparecen.

En el PIDE del periodo 1995-1997 destaca la necesidad de incrementar la cobertura promocionando carreras con una reducida matrícula para mantener la estabilidad del crecimiento. Para subsanar el problema, el plan consideró contener el crecimiento en las áreas sociales y administrativas, y generar un proceso de reorientación de la matrícula a las áreas prioritarias como las ciencias agropecuarias, naturales y exactas, e ingeniería.

Para el PIDE 1998-2001, titulado Estrategia Horizonte Siglo XXI, se puede observar una clara alineación de las acciones propuestas con el Plan Nacional de Desarrollo 1995-2000 de aumentar la cobertura considerando la apertura del mayor número posible de oportunidades educativas, con base en criterios de calidad. Lo planteado se presenta como si el estudiantado no tuviera ni género, ni sexo, es decir, desdibujando que la población estudiantil está compuesta por hombres y mujeres, planteando a manera de solución, una selección equitativa pero que no reconoce una trayectoria que ha sido inequitativa, presentando una ausencia considerable de perspectiva de género.

Para el diseño del PIDE 2002-2005, se utilizaron las líneas estratégicas de la Asociación Nacional de Universidades e Instituciones de Educación Superior (ANUIES), así como los postulados de equidad y humanismo, con lo que se buscaba que el Subsistema de Educación Superior tuviera atención a la equidad. Esta alineación a lo propuesto en ANUIES y al Programa Nacional de Educación hace que los aspectos de equidad de género queden considerados de manera igualitaria entre los "grupos vulnerables" o desfavorecidos, aun cuando cada uno de los mencionados requiere de esfuerzos y estrategias distintas. $\mathrm{Al}$ reunirlos de forma simplificada se minimiza el problema, provocando que las acciones para disminuir la inequidad de género sean dispersas y por tanto de bajo impacto. Son estos los riesgos de la alineación de las acciones de la universidad con los programas públicos, pues las omisiones cometidas en el diseño de los planes y programas nacionales se decantan en las instituciones que las implementan.

El PIDE 2006-2009 continúa con los postulados orientadores de ANUIES de: fortalecer las estrategias y acciones de atención a los estudiantes, considerándolos el eje central de los procesos académicos; fortalecer el programa de becas, prestando especial atención a los estudiantes con mayores necesidades económicas y a los principios de equidad para la permanencia, como si la razón principal de la deserción de los alumnos fuera exclusivamente por aspectos financieros, cuando para la equidad de género existen más factores, además del económico.

Es hasta el Plan Institucional de Desarrollo 20102013, que se presenta como parte de la formación de los estudiantes, el tema de equidad de género, mediante un conjunto de acciones que promueven el respeto a la diversidad, la equidad de género (PIDE 2010-2013). La inclusión de la "equidad de género" fue acompañada del "respeto a la diversidad", dos términos que en últimas fechas se utilizaran como sinónimos, e incluso se considera que la entrada en la agenda de los temas de diversidad desplazó en importancia y prioridad a los temas de género.

El PIDE 2014-2017, denominado "Educación con responsabilidad social", propone la igualdad de género, junto con la promoción de la salud, y el establecimiento de estilos de vida sostenibles. Es importante 
destacar que en este documento se abordan por primera vez los aspectos laborales de equidad de género, pues en planes institucionales anteriores sólo hacía referencia al alumnado, mientras que en este último se propone como parte del desarrollo del personal de la Universidad.

Así, los documentos oficiales de planeación institucional pasaron de una "equidad de género" a una "igualdad de género" sin cuestionarse el cambio o utilizadas como si fueran sinónimos, es decir, sin tomar en cuenta que para que exista una verdadera "igualdad de género" es necesaria e indispensable una "equidad de género".

En el análisis condensado de los planes institucionales, se puede identificar claramente que los temas de género no se encuentran como puntos principales de la agenda y que cuando se incluyen se ponen en la misma jerarquía de los "grupos vulnerables" o sus estrategias no son claras en términos de que efectivamente reducirán la discriminación y las inequidades hacía las mujeres, tanto en ámbitos del alumnado como del personal académico y directivo.

Para Lamas (2000), la clasificación cultural del género define no sólo la división del trabajo, las prácticas rituales y el ejercicio de poder, sino que se atribuyen características exclusivas a uno y otro sexo en materia de moral, psicología y afectividad. La cultura marca a los sexos con el género y el género marca la percepción de todo lo demás: lo social, lo político, lo religioso, lo cotidiano. Los aspectos por investigar serían si estas omisiones en los planes y la carencia de sustento en las estrategias son una forma de mantener el orden patriarcal establecido en la cultura de la institución, además de representar un desconocimiento de los temas de género.

\section{EI PIFI: un prepuesto para la equidad de género}

El Programa Integral de Fortalecimiento Institucional (PIFI) fue una estrategia de la SEP para apoyar a las instituciones de educación superior a lograr mejores niveles de calidad en sus programas educativos y servicios que ofrecen. A través de este programa, las instituciones recibían recursos en respuesta a las prioridades que derivarían de un ejercicio de planeación estratégica participativa concursables anualmente (no regularizables). Este programa de apoyos ha continuado, pero su nombre ha variado por la alternancia en el gobierno federal, aunque conserva mucho de sus origenes.

La reglas de operación de los proyectos PIFI especifican que las instituciones propondrán un proyecto transversal (ProGES) donde se incorporará un proyecto para "Fomentar la perspectiva de género", al que se otorgará el $4.47 \%$ del total de los recursos otorgados a la institución para fomentar la cultura de la perspectiva de género. Los recursos que se autoricen para cada proyecto sólo podrán destinarse a cualquiera de las siguientes cinco acciones en materia de perspectiva de género (SEP, 2012): 1) Diseñar e implementar un Plan de Capacitación para el personal y/o estudiantes. 2) Formular un estudio al interior de la institución. 3) Diseñar y difundir una campaña de sensibilización. 4) Adquirir material bibliohemerográfico sobre el tema. 5) Elaborar y publicar libros arbitrados en torno al tema.

La institución analizada obtuvo en el año 2012 un recurso por $\$ 368450$ para el proyecto titulado: Cultura Universitaria para la Igualdad de Género, del que se desprende el primer objetivo: Consolidar el programa cultural institucional con perspectiva de género en procesos de sensibilización dirigido a personal directivo, docente, mandos medios, administrativos y de servicios. En el desglose de los recursos de la reprogramación para el objetivo 1, se propone como acción relevante el pago de un libro como resultado de proyectos de investigación con perspectiva de género y su difusión (\$72 300), sin embargo en este mismo rubro se propone la edición de un libro (\$18 616) que no corresponde a temas sobre género, sino que trata de filosofía de las ciencias y estética. También se especifica una campaña institucional: 
"Ni más ni menos. Cultura Universitaria para la Igualdad de Género" (\$16 530) para la impresión de separadores de libros, pendones, un video y cápsulas de radio.

Destaca de este objetivo particular la poca coincidencia de lo propuesto en el proyecto institucional con lo jerarquizado en los lineamientos del PIFI, pues la campaña de sensibilización fue de prioridad baja, mientras que los estudios de género, así como los planes de capacitación considerados de mayor importancia e impacto no se contemplan en el proyecto. A esto se suman rubros como la edición de un libro que no tendrá repercusiones en la comunidad en general, respecto a la equidad de género.

En el segundo objetivo: Actualizar la Biblioteca Virtual de Género de la Universidad (\$189 906), donde se busca cumplir una meta de incrementar en 150 recursos anuales el acervo digital de la Biblioteca Virtual de Género, se propone una lista de libros. Para el análisis de los acervos propuestos se buscó la reseña de algunos de ellos en páginas de internet para tener una aproximación del contenido: la conclusión fue que la elección de los títulos se hizo entrar con "calzador" al proyecto y no abonan a los objetivos propuestos en las reglas de Operación del PIFI. Entre los títulos adquiridos destacan los siguientes: ${ }_{i} M i$ bebé o mi jefe? Cómo conciliar la vida familiar con el trabajo (Educación y Pedagogía), Caballeros, bribones y pájaros egocéntricos (Matemáticas recreativas), La construcción de la lesbiana perversa (Comunicación-biografias), Consultorio sexual para todas las especies (Biología), Disfunciones sexuales femeninas (Psicología clínica), Fabricando bebés ¿Existe un derecho a tener hijos? (Biología-bioética). Otros acervos propuestos versan sobre temas de terapia familiar, sexualidad y preferencias sexuales, amor, autoestima y salud. Es posible notar que este conjunto de textos no pertenecen al acervo de una "Biblioteca de Género" y que por lo tanto no hubo una aportación relevante al tema.

El proyecto presentado no muestra una articulación entre las acciones, ni tampoco un apego a la priorización propuesta en las reglas de operación de los proyectos PIFI en la materia. Resulta necesario un diagnóstico sobre la situación que guarda la equidad de género, que indique cuál es el punto de partida, y determine la priorización de las brechas a resolver mediante un esquema real de capacitación, que esté claramente definido hacia lo que la universidad desea lograr en su agenda de género y por sobre todo que permee a toda la comunidad universitaria.

\section{El reglamento escolar}

Para el análisis del reglamento escolar de educación superior vigente en la institución (presentado en el acuerdo 4 de 2006) se parte de la selección de algunos artículos que se consideraron importantes para facilitar la equidad de género en el ingreso, permanencia y egreso.

En lo que respecta al proceso de admisión, el artículo tercero menciona que se aceptará a los aspirantes que cumplan con los criterios de admisión vigentes, siempre y cuando se disponga de la capacidad de atención respecto a la infraestructura, planta docente y administrativa en cada plantel. Por lo tanto, se infiere que la institución considera a los aspirantes en igualdad de condiciones, es decir, los hombres y las mujeres afrontaron las mismas situaciones o tuvieron las mismas oportunidades para terminar el bachillerato e iniciar una carrera. Situación que se repite con lo plasmado en los planes institucionales.

Sobre la permanencia en la institución, en el Capítulo III "De la asistencia a las actividades académicas" (artículos 44 y 47), se hace énfasis en la asistencia como necesaria para permanecer en la carrera; los alumnos tienen la obligación de presentarse puntualmente a la hora indicada para empezar el trabajo académico y, en caso de enfermedad, los alumnos deberán acreditar su incapacidad, en un plazo no mayor de 72 horas. En este aspecto, el reglamento no considera los tiempos necesarios - solamente los que se le otorguen de incapacidad - para etapas de embarazo, ni de lactancia de las mujeres que optan 
por ser madres durante la carrera; tampoco se consideran los tiempos para los hombres que deseen apoyar a sus parejas durante los días cercanos al parto. Se entiende entonces que para terminar una carrera universitaria, se debe diferir la etapa de embarazo, porque no está contemplada en el reglamento y por lo tanto, en caso de decidir un embarazo se arriesga de manera considerable la permanencia en la institución.

En lo relativo a los aspectos de orden y disciplina en las instalaciones universitarias, el artículo 52 estipula que las medidas o sanciones disciplinarias se aplicarán cuando el alumno falte al respeto a maestros, compañeros, trabajadores y autoridades universitarias. Sin embargo, no se especifica sobre faltas que tengan que ver con aspectos de equidad de género, quedan de manera ambigua y subjetiva en una "falta de respeto" que al ser evaluada por un comité con poca sensibilidad hacia aspectos de género, minimizaría el tema y lo consideraría una exageración por parte de quien denuncia un abuso.

Considerando que el reglamento regula la vida cotidiana tanto entre el alumnado como en la relación alumno-docente, resulta necesaria una propuesta que contemple aspectos que hagan efectivamente posible una equidad de género, en la que se proponga un porcentaje mínimo de la matrícula de ingreso tanto para los hombres en carreras feminizadas y de mujeres en carreras masculinizadas. A la par del reglamento, es necesario que se incorpore apoyo a mujeres embarazadas y a sus parejas, mediante un esquema de becas y apoyo psicológico para afrontar los cambios y nuevas responsabilidades.

\section{Darle voz al género: la interpretación de "lo dicho"}

A través del testimonio confidencial de los funcionarios entrevistados relacionados con las áreas docentes y estudiantiles se comprobó que en la institución no existe actualmente un programa específico que sea responsable de promover acciones o de dar seguimiento a normativas relacionadas con la equidad de género, la discriminación y la violencia de género al interior de la institución. Los entrevistados reconocen que se vive un contexto social crítico, duro en el país, que tiene gran impacto en la comunidad universitaria, sin distinción de alguna área en particular. Por razones que van desde la voluntad política, hasta tiempo y recursos económicos, no se ha podido implementar un programa de este tipo. Como resultado del análisis de las entrevistas y partiendo de su nivel interpretativo, los resultados se pueden agrupar en los siguientes apartados:

\section{a) Equidad}

De acuerdo con los informantes, la preocupación por temas como la violencia de género es reciente en la institución, y lo que se ha trabajado es en el nivel de investigaciones de cuerpos académicos relacionadas con el tema, sin llegar a influir en el nivel institucional para la toma de decisiones.

Esas son cosas recientes de las preocupaciones por la violencia de género, pero no se puede decir que sea una preocupación que surge en el nivel institucional. Son inquietudes de personas que trabajan o que colaboran en la universidad y normalmente mujeres (Informante 1).

Si bien no existen cifras ni datos de los temas de este estudio en la universidad, la cercanía que tienen los informantes con la comunidad universitaria nos permite conocer los casos frecuentes que se dan en el entorno cotidiano.

La percepción que tienen los informantes sobre la equidad de género es que sólo está en el discurso y no ha ido más allá. Se ha trabajado por la equidad social, por dar oportunidades a los que menos tienen, pero no por ser de un género.

El fomento de la equidad de género está, pero no pasa del discurso. Cada vez que llega el 8 de marzo y 
hay que hablar de la participación de las mujeres, se habla de la equidad de género (Informante 1).

La génesis del problema está desde la definición de "equidad": en el término "equidad" quedan incluidas muchas formas que no son la de género, por ejemplo, equidad social, porque habría que promover a los grupos más desfavorecidos, porque ellos están en una situación vulnerable. Entonces es "equidad" en el término social más amplio, o hacia los grupos con discapacidad, o hacia la diversidad sexual. Entonces, sobre el paraguas de la equidad, caen muchas cosas (Informante 1).

En los discursos que informan sobre los avances en materia de equidad en la universidad, se engloba esta serie de componentes que en ocasiones resultan ambiguos aunque se traten como iguales:

Entonces hablan de equidad y a veces parece que si se refieren a la de género y a veces no, pero para efectos reales y operativos, las acciones a favor de las mujeres ha habido muy pocas. Yo creo que en la Universidad $[\ldots]$, la percepción que tenemos es que eso no ha cambiado (Informante 1).

En el aspecto laboral, por ejemplo se perciben también posibles casos de inequidad, pues es difícil otorgar puestos directivos a mujeres, aun cuando conste su preparación.

Para los casos de inequidad de género hay mujeres a las cuales se les ha dilatado o postergado el otorgamiento de un determinado nombramiento y uno supone que puede haber ahí una cuestión de género. Finalmente la persona se queda en calidad de suplente o sustituto por un periodo muy prolongado, sacando el trabajo y luego "zas" le ponen un jefe, que normalmente es varón (Informante 1).

En este tenor, se obvian aspectos basados en el desconocimiento de lo que es la equidad de género, y se considera que las cosas están bien tal y como están, con niveles cercanos a la perfección y por consecuencia no se proponen estrategias que fomenten la equidad de género.

Antes, cuando se hablaba de equidad se asumía que tenía que ver con las diferencias entre los que tienen y los que están menos favorecidos, a últimas fechas cuando empieza a hablarse a nivel central de programas de equidad de género [en la institución] decimos "nosotros ya lo hacemos" porque nos preocupamos por la equidad, pero en realidad no es equidad de género (Informante 1).

Cabe señalar que se incorporan nuevos temas a la agenda, como el respeto a la diversidad sexual y la disminución de la violencia hacía estos grupos, aspecto que resulta necesario y pertinente de atender, pero que al formar parte de la misma definición de equidad, redunda en diluir los esfuerzos y avances en la equidad de género:

La diversidad sexual es una línea de moda, que toma preminencia y parece tener una gran aceptación en todos lados, entonces hay una especie de soslayamiento hacia el feminismo, porque ahora parece que lo de moda es el tema de la diversidad por sobre el de feminismo. Aun cuando el asunto de los derechos de las mujeres abarca a la mitad de la población y las personas cuya opción sexual es la "no convencional" podríamos pensar que son un 10 o un $15 \%$, es una proporción menor, sin embargo ha tenido mucha visibilidad y mucho apoyo. Tengo la impresión de que para la gente es políticamente correcto ser open mind y está desplazando a los estudios, apoyos y visibilidad de los temas de feminismo (Informante 1).

Resulta necesario, indispensable y urgente definir en su contexto y realidad la equidad de género en la universidad y los componentes que la conforman. 
Una vez clarificado el término para las autoridades, proponer políticas y lineamientos jerarquizados que promuevan un cambio que se legitime en la vida cotidiana institucional.

\section{b) Violencia y discriminación}

De acuerdo con el testimonio de los informantes, en la institución se ha dado un incremento en la violencia, principalmente entre alumnos. Lo más frecuente ha sido la violencia entre parejas de novios, desde la psicológica y emocional, hasta la física.

Hay alumnas golpeadas por alumnos, compañeras de trabajo que llegan golpeadas con lentes obscuros, la interacción entre los alumnos de manera verbal se ha deteriorado mucho, también existe el acoso del jefe, como forma de violencia que no se dice porque les costaría el puesto [...] Una persona hace algunos años vino aquí llorando y me dijo: "No pida que me cambien, que no me vayan a cambiar, porque me están pidiendo que me vaya a tal parte, pero es con esta intención, entonces por favor pelee que yo me quede aquí, porque no me quiero ir". Y así te puedo decir muchos casos a lo largo del tiempo que llevo aquí. Predominantemente de hombres hacía mujeres (Informante 1).

Algunas piden ayuda pero la mayoría "No quieren evidenciarse y sobre todo, no quieren evidenciar a la pareja" (Informante 2). Por lo tanto, pocos buscan apoyo profesional. Quienes llegan a manifestarlo reciben un acompañamiento, o se han canalizado a lugares especializados:

En muchas ocasiones lo que pedimos es la asesoría al abogado general, para que a su vez también asesore a las personas, que en un momento dado quisieran establecer una denuncia" (Informante 2).

Respecto a la discriminación, esta es menos visibilizada por las personas entrevistadas al afirmar que forma parte del bullying entre el alumnado, donde el trato discriminatorio por cuestión de género o de sexo es cuando específicamente se burlan de personas por sus características y preferencias. La solución se da en el salón de clase, solicitando respeto entre compañeros. Sin embargo, en lo laboral también se presenta un fenómeno de acoso:

El otro día alguien me decía 'es que no puede ser que $\mathrm{X}$ y $\mathrm{Y}$, dos personas, se estén besando en la oficina’. Ok, está mal que se estén besando, pero parecía que lo más escandaloso es que se tratara de una pareja homosexual (Informante 1).

Otro tipo de discriminación se detecta hacia las mujeres que ocupan cargos directivos. Mediante la descalificación, rumores indirectos y etiquetas, perjudican su imagen cuando no se comporta como ellos esperarían.

Los casos de discriminación hacia las directoras, ya sea por la personalidad, concentradas en el trabajo, no sales con nadie, la manera de ser tan directa, se les tipifica de homosexuales. La gente habla de las directoras por el hecho de no tener pareja y hay un cierto rechazo (Informante 1).

Respecto a las necesidades que detectan los informantes, para aplicar con los alumnos que son víctimas de violencia y discriminación, se habla de la creación de un protocolo de atención. "Muchos directores actúan con cierto sentido común $[\ldots]$ y necesitamos saber qué hacer con ellos, cómo trabajar y no nada más aplicar en un momento dado un reglamento de expulsión" (Informante 2). El entrevistado menciona también como gran necesidad la educación emocional para lograr una formación integral que impacte en la actitud individual y poder acabar con la ira, el coraje y la envidia que percibe en los estudiantes. Afirma que ello tendría una repercusión importante en la violencia. 
Por otra parte llama la atención el dato de que no cuentan con ningún tipo de capacitación con perspectiva de género para los orientadores vocacionales y no entra en las necesidades del programa. Se ubica al Programa de Estudios de Género como una instancia que contribuye, sin embargo, se desconocen las acciones específicas que lleva a cabo.

También se afirma que hay algunas dependencias que "le apuestan a esto" a través de cursos y diplomados - no siempre específicos-, que pueden incluir la temática y fomentar en los asistentes el respeto.

Se mencionó que en la red centro-occidente del programa de Tutoría, por ejemplo, se ventilan problemáticas que son muy semejantes y es a través de comentarios sobre la forma en que cada quien resuelve las cosas, con un cierto sentido común, como se toman decisiones. Esto evidencia la necesidad de contar con documentos formales.

Resultan también necesarios los protocolos para el entorno laboral, para atender los casos de las trabajadoras universitarias, ajustar los reglamentos de trabajo para evitar acoso, violencia de género y discriminación por género. De ellos se esperaría que las conductas no deseadas sean sancionadas para disminuir la problemática, pero que a la par exista un esquema de intervención adecuado a las víctimas.

\section{c) Los proyectos}

Para plantear los proyectos institucionales de fomento a la equidad de género se puede aprovechar lo que ocurre en el contexto, donde ciertos "grupos muy cargados políticamente", piden que se declare en el estado la alerta de género, aunque existe otro grupo de fuerzas políticas que dificulta emprender acciones:

Parece que a los gobiernos no les conviene eso, por lo tanto el proceso para declararla resulta sumamente difícil, tienen que reunirse tantos requisitos y tantas cosas, que parece imposible (Informante 1).

A este respecto, en el interior de la institución se afirma que existe la sensibilidad de algunas universitarias por hacer algo, dado el aumento de feminicidios donde se han involucrado estudiantes, sin embargo, los tiempos y recursos solo permitirán hacer una serie de recomendaciones para incluir en el programa de Calidad de Vida.

Un grupo de universitarias nos reunimos en torno al tema del incremento de feminicidios con casos de estudiantes y demás, pero hay una especie de silencio institucional al respecto, y entonces nosotras estába-

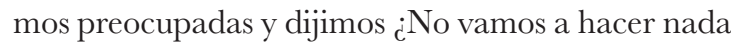
como mujeres, como universitarias? (Informante 1). ${ }^{2}$

Esta informante considera que debe haber apoyo desde el área de liderazgo de la institución, tener esa intención, para poder incidir significativamente, pues no pueden ser iniciativas aisladas:

Es poco lo que podemos hacer, porque nuestro tiempo, nuestras actividades se consumen en otras cosas. Nos puede preocupar el tema, pero difícilmente podemos ir más allá (Informante 1).

Además, es necesario que lo propuesto en los proyectos sea coherente y exista un compromiso de los directivos, pues los proyectos del programa PIFI para promover la equidad de género en la institución presentaban inconsistencias:

La universidad también generó su proyecto. Pero la intención de la SEP era que los altos funcionarios se sensibilizaran en torno a esto y entonces se organizan los eventos y acaban yendo los estudiantes. Los funcionarios nunca van, son reactivos, pero institucionalmente se cumple, porque los parámetros para el

\footnotetext{
${ }^{2}$ Posterior a haberse realizado esta entrevista, fue activada la alerta de género en el estado.
} 
PIFI dicen "cuántas acciones se realizaron” y debería ser "qué cosas cambiaron/modificaron" a partir de la implementación de este programa (Informante 1).

Los resultados no fueron los mejores. Se cumplieron las metas, pero la gente que debería sensibilizarse - como los altos funcionarios-, no se involucraron. Entonces no representan cambios significativos a las dinámicas y la cultura institucional:

Estamos en la línea, pero no vamos más allá, no hay un convencimiento real de decir "estamos queriendo hacer esto, ha llegado ese dinero, aprovechémoslo porque queremos cambiar", y no, nos llega ese dinero y "a ver en que nos lo gastamos" (Informante 1).

En proyectos presentados en otras instituciones la situación parece complicarse aún más:

Me tocó participar como evaluador de esos proyectos y hubo cosas escandalosas, por ejemplo, una institución que contrató a un despacho de contadores para hacer el programa de género. Entonces todo consistía en cursos y cursos y cursos [...] Había unas propuestas muy buenas [...], pero el impacto siempre va a depender del liderazgo en las instituciones. También las esposas de los rectores son muy importantes: el rector de la Autónoma de Sinaloa estaba casado con una feminista, entonces, usando ese dinerito del PIFI impulsaron un montón de acciones que sí tenían impacto, que sí generaron cambios, ¿por qué? porque desde el área de liderazgo de la institución, había esa intención (Informante 1).

Para que estos proyectos funcionen, por lo tanto, debe existir alguien en los altos niveles que conozca sobre el tema y haga propuestas que efectivamente impacten a la equidad de género; que sea un proyecto significativo que verdaderamente busque cambios a la cultura institucional y no únicamente se logre la ejecución de recursos federales.

\section{A manera de cierre}

Ante estas situaciones los temas de equidad de género en la institución analizada - reflejo de muchas otras - , coinciden con lo mencionado por Lagarde (2011), donde la mayoría de las mujeres vive, por lo menos, con dos calendarios vitales: el culturalmente aceptado para su sociedad y el suyo; el culturalmente aceptado, dictado desde una perspectiva patriarcal en donde para las mujeres su actitud vital es la espera social, mientras que para el hombre está reservada la trascendencia mediante su realización protagónica en la vida.

A pesar de que en los últimos años se han planteado políticas para mejorar la equidad en las universidades (Fuentes, 2016), los que toman decisiones las desconocen y no adoptan las medidas necesarias, como se ha podido constatar ahora.

Se debe trabajar fuertemente en una intervención que modifique el imaginario en los directivos de la universidad pública que minimiza las injusticias de género y redunda en la simulación y el engaño colectivo respecto al tema. Así se concibe que la equidad de género se mejora modificando mínimamente algunos esquemas y las buenas voluntades; las dinámicas y la cultura institucional cambian y avanzan de forma considerable, lo cual no ocurre. En estos intentos, los y las activistas se desgastan ante el "aquí no pasa nada". Lo anterior resulta indispensable pues se reconoce que es desde los altos niveles jerárquicos que se deben apoyar y conjuntar las iniciativas de grupos aislados y el diseño de políticas y programas jerarquizados de manera adecuada a la problemática y realidad institucional.

El análisis de documentos institucionales, así como de los informes y planes institucionales, dan una guía tanto en sus omisiones como en las cosas que se dejan como obvias. En los PIDE, los aspectos de equidad de género se introducen vagamente, desde la perspectiva de grupo vulnerable, siguiendo la línea de la ANUIES, pero no existe una iniciativa institucional. 
Resulta indispensable que la elaboración de los futuros planes institucionales consideren en su agenda de género los tres momentos considerados por Aguilar (2009), que si bien están pensados para las políticas públicas, aplican a las políticas y programas institucionales: la creación de la política: donde compiten los intereses particulares y grupales de los sujetos y grupos de poder; la implementación: la conversión de estas políticas en programas con presupuesto, tiempo, metas a alcanzar y la evaluación: realizada para los informes anuales. Cabe señalar que se debe prestar especial atención a la implementación de los planes y programas, porque es donde más se desvirtúa lo planeado, donde se originan las consecuencias no deseadas, no planeadas y no esperadas, que más que beneficiar, afectan el desarrollo de una verdadera cultura de equidad de género sobre la que se busca el impacto.

Los investigadores sobre temas de género en las instituciones educativas de este nivel son los que podrían definir una agenda y proponer acciones que obedezcan al contexto actual, en el que se tome en consideración el pasado y el presente institucional, lo que permitirá estrategias de intervención que no partan de cero. También es necesario que los resultados de diagnósticos de las oportunidades educativas para desarrollar la transformación de género sean aprovechados mientras resulten actuales y pertinentes, pues con el paso del tiempo se convierten en letra muerta o en acciones que se impulsan de forma aislada por pequeños grupos interesados en el tema de la equidad de género. Precisamente Martínez y Kral (2008) proponen: 1) Dar continuidad al análisis del acceso de las mujeres a la educación superior; 2) Diversificar las oportunidades mediante modalidades abiertas, flexibles y a distancia; 3) Apoyar la retención escolar de madres y padres adolescentes y jóvenes; 4) Aumentar la incorporación de las mujeres al posgrado; 5) Crear un comité institucional en pro de la equidad de género y la integración de un observatorio; 6) Incorporar el enfoque de género en las instituciones de educación superior.

Se debe tener presente la vigilancia para verificar que los planes, programas y acciones promuevan efectivamente una equidad de género y no algo que perpetúe los roles de género, como es el caso de acciones de mujeres incorporadas al voluntariado realizando trabajo social y altruista, en el que el rol maternal y de cuidado se hegemoniza y los buenos sentimientos son virtudes femeninas y no masculinas. ¿Por qué no existe un voluntariado similar formado por un grupo de caballeros y esposos de directivas de la institución? (Acuña, 2008).

Con respecto a los proyectos que otorgan recursos específicos para equidad de género a las instituciones se puede afirmar que han sido muy pocos. Uno de ellos ha sido a través del PIFI, en el que la universidad deberá analizar a fondo y de manera crítica lo que se proponga en estos proyectos, pensándolos en esquemas de largo aliento, para que repercutan en modificar la cultura y el estilo institucional; en donde las propuestas redunden en generar mejoras a los programas educativos, lineamientos y políticas para el diseño curricular, reglamento escolar y esquemas de apoyo institucionales que consideren la perspectiva de género.

En las políticas públicas federales para la educación superior, así como en la institución analizada, la equidad de género aparece como trinomio inseparable: equidad, violencia, discriminación. Sin embargo el problema es más complejo, pues cada una de ellas requiere de intervenciones distintas, aun cuando el discurso político las considera como un todo. Esta situación debe abordarse desde sus distintas aristas tanto teóricas como metodológicas. Es necesario recalcar que la inconsistencia está en la cantidad de recursos asignados en los programas para atender el trinomio mencionado. 


\section{Referencias}

Acuña, M. (2008), "La participación femenina en los proyectos universitarios", en En busca de la equidad de género en la universidad, México, Universidad de Colima, pp. 189-206.

Aguilar, L. (2009), "Marco para el análisis de las políticas públicas", en Política pública y democracia en América Latina. Del Análisis a la implementación, México, Miguel Ángel Porrúa, pp.11-31.

Eisenstein, Z. (1999), "La pedagogía jurídica como silencio o silencios autorizados", en Feminismosy pedagogías en la vida cotidiana, Madrid, Morata, pp. 243-258.

Elias, N. (2009), El proceso de la civilización: investigaciones sociogenéticas y psicogenéticas, México, FCE.

Fernández, L. (2005), Instituciones educativas: dinámicas institucionales en situaciones críticas, Buenos Aires, Paidós

Foucault, M. (2010), Michel Foucault. Obras esenciales, España, Paidós.

Fuentes Vásquez, L. Y. (2016), “Por qué se requieren políticas de equidad de género en la educación superior?", en Nómadas (Col), (44), <http://www. redalyc.org/pdf/1051/105146818005.pdf> [Consulta: febrero de 2017].

Geertz, C. (2005), La interpretación de las culturas, Barcelona, Gedisa.

Goffman, E. (2012), La presentación de la persona en la vida cotidiana, Buenos Aires, Amorrortu.

Iñiguez, L. (2003), Análisis del discurso. Manual para las ciencias sociales, Barcelona, UOC.

Lagarde, M. (2011), Los cautiverios de las mujeres: madresposas, monjas, putas, presas y locas, México, UNAM.

Lamas, M. (2000), "Diferencias de sexo, género y diferencia sexual", en Cuicuilco, enero-abril, vol. 7, número 18, <http://www.redalyc.org/pdf/351/35101807. pdf> [Consulta: agosto de 2017].

Lee, S. (1999), "La pedagogía de la vergüenza", en Feminismos y pedagogías en la vida cotidiana, Madrid, Morata, pp. 211-224.

Lourau, R. (2001), El análisis institucional, Buenos Aires, Amorrortu.
Luke, C. (1999), Feminismos y pedagogías en la vida cotidiana, Madrid, Morata.

Martínez, S. (2006), Mujeres y universidad: vidas académicas, México, Universidad de Colima.

Martínez, S. y K. Kral (2008), "La conciencia transformadora de género en la universidad", en En busca de la equidad de género en la universidad, México, Universidad de Colima, pp. 95-123.

Miranda, R. (2007), "Mujeres, educación superior e igualdad de género", en CPU-eRevista de Investigación Educativa, núm. 4, enero-junio, <http://www.redalyc. org/pdf/2831/283121710004.pdf> [Consulta: agosto de 2017].

Monroy, C. E., G. Tapia, L. García y S. López (2010), Reseña Histórica de la Universidad de Colima, Colima, Universidad de Colima.

Morgan, D. (1999), "Aprender a ser hombre: problemas y contradicciones de la experiencia masculina", en Feminismos y pedagogías en la vida cotidiana, Madrid, Morata, pp. 106-116.

Palomar, C. (2011), La cultura institucional de género en la Universidad de Guadalajara, México, ANUIES.

Rodríguez, M. (2008), "Historia de la participación de las mujeres en la Universidad de Colima (1940-1980)", en En busca de la equidad de género en la Universidad, un estudio de caso, México, Universidad de Colima, pp. 69-91.

Remedi, E. (2004), Instituciones educativas: sujetos, historia e identidades, México, Plaza y Valdés.

Ruiz-Ramírez, R. y M. R. Ayala-Carillo (2016), "Violencia de género en instituciones de educación", en Ra Ximhai, enero-junio, pp. 21-32, <http://www.redalyc.org/ html/461/46146696002/> [Consulta: agosto de 2017].

Secretaría de Educación Pública (SEP) (2012), Reglas de operación de PIFI 2012, <http://pifi.sep.gob.mx/pifi/ reglas/2012/ro_pifi_2012.pdf> [Consulta: agosto de $2017]$.

Van Dijk, T. (2002), "El análisis crítico del discurso y el pensamiento social", en Athenea Digital. Revista de Pensamiento e Investigación Social, núm. 1, primavera, pp. 18-24. 
Wodak, Ruth y Michel Meyer (2003), Métodos de análisis crítico del discurso, Barcelona, Gedisa.

\section{Documentos Institucionales:}

- Informe Rectoría 2016

- Plan Institucional de Desarrollo 1995-1997.

- Plan Institucional de Desarrollo 1998-2001.

- Plan Institucional de Desarrollo 2002-2005.
- Plan Institucional de Desarrollo 2006-2009.

- Plan Institucional de Desarrollo 2010-2013 Visión 2030, Universidad sin Fronteras.

- Plan Institucional de Desarrollo 2014-2017 (PIDE).

- Proyecto PIFI institucional 2012 (Aplicado en 2013 y 2014).

- Reglamento Escolar. pública estatal: discrepancias institucionales", en Revista Iberoamericana de Educación Superior (RIES), México, UNAM-IISUE/Universia, vol. IX, núm. 25, pp. 138-156, http://dx.doi.org/10.22201/iisue.20072872e.2019.25.346 [consulta: fecha de última consulta]. 